\title{
Análise da Demanda de Energia Elétrica no Setor Industrial no Brasil
}

Márcio Margelo Gross

(Economista na Universidade Federal de Santa Maria - UFSM)

Claílton Ataídes de Freitas

(Professor no Departamento de Ciências Econômicas e Programa de Pós-Graduação em Economia e Desenvolvimento da Universidade Federal de Santa Maria - PPGE\&D/UFSM)

Cezar Augusto Pereira dos Santos

(Professor na Universidade Comunitária da Região de Chapecó - UNOCHAPECÓ)

Dieison Lenon Casagrande

(Doutorando no Programa de Pós-Graduação em Economia da Universidade Federal de Pernambuco PIMES/UFPE)

Paulo Henrique Hoeckel

(Doutorando no Programa de Pós-Graduação em Economia do Desenvolvimento da Pontifícia Universidade Católica do Rio Grande do Sul - PPGE/PUCRS)

\begin{abstract}
Resumo:
O presente artigo tem por objetivo estimar as relações de longo e curto prazo da demanda por energia elétrica do setor industrial do Brasil através de um Modelo de Auto-Regressão Vetorial com correção de erro (VECM), pelo método de Co-integração de Johansen, para assim determinar as elasticidades renda, preço da demanda e preço cruzado por energia elétrica. Objetiva-se ainda projetar a demanda por energia elétrica para os meses de junho, julho e agosto de 2012 através da metodologia Box-Jenkins. Os resultados encontrados apontam que as elasticidades estão de acordo com a teoria econômica e com os resultados encontrados na literatura. A energia se mostrou um bem normal, com demanda inelástica e um bem substituto em relação ao petróleo para as indústrias que possuem essa mobilidade. $\mathrm{O}$ modelo de previsão que apresentou melhor ajustamento aos dados foi um modelo AR $(4,12)$ MA $(4,12)$. A média da exatidão das previsões realizadas foi de $97,46 \%$, que é considerado um bom grau de ajustamento, considerando o fato de os dados serem mensais, onde as oscilações são mais difíceis de serem previstas. Esses resultados revelam que é possível utilizar dados mensais para previsões de curto prazo em modelagens Box-Jenkins.
\end{abstract}

Palavras-Chaves: demanda por energia elétrica; Modelo VECM; Metodologia Box-Jenkins.

\begin{abstract}
:
This paper aims to estimate the relationship of long and short-term demand for electricity in the industrial sector in Brazil through an Auto-Regression Model Vector Error Correction (VECM), at method of Johansen Co-integration, well to determine the income elasticity, price demand and price crossed by electricity. Another goal is to project the demand for electricity for the months of June, July and August of 2012 through the Box-Jenkins methodology. The results show that the elasticities are consistent with economic theory and with the results found in the literature. The energy showed a normal good with inelastic demand and a good substitute for oil for industries that have such mobility. The forecasting model that showed the best fit to the data was a model AR $(4,12)$ MA $(4,12)$. The average accuracy of the predictions made was $97,46 \%$, which is considered a good degree of adjustment, considering the fact the data is monthly, where the oscillations are more difficult to predict. These results show that it is possible to use monthly data for short-term forecasts in Box-Jenkins modeling.
\end{abstract}

Keywords: demand for electricity; VECM model; Box-Jenkins methodology. 


\section{1) INTRODUÇÃO}

Ao acender uma lâmpada, ligar um notebook, subir num elevador e para a maioria das atividades de nosso cotidiano a energia elétrica está presente. É difícil imaginar o dia-a-dia sem ela, tanto por parte do cidadão comum quanto no caso das empresas, que para se manter em atividade, dependem de energia, em sua maioria, a elétrica.

A energia elétrica é uma das principais fontes que compõem a matriz energética brasileira. Sua geração provém em sua maioria de hidroelétricas (IBGE, 2012). Assim, conhecer a sua demanda é importante tanto para políticas públicas como estratégicas. Como a energia é um dos pilares de sustentação da produção econômica de um país, estudos relativos a essa temática são abrangentes.

Dentre estudos recentes, convergentes com a temática proposta neste artigo, destacam-se: Schmidt e Lima (2004), Siqueira e Hollanda (2005), Mattos (2005) e Irffi et al. (2009). Es77ses autores analisaram e estimaram a demanda por energia elétrica no setor industrial, além de estimarem também a demanda residencial e comercial. Andrade e Lobão (1997) analisaram apenas o setor residencial. Castro e Rosental (2008) estimaram as elasticidades para o Brasil de forma agregada. Leite (2006) e EPE (2011) projetaram a demanda de forma agregada para o Brasil. Nestes estudos, além de buscas por simulações de previsão de demanda desse tipo de energia no setor industrial, foi analisada, em termos conjunturais, a dinâmica regional do consumo de energia elétrica.

Sabe-se que a indústria responde por 14,6\% do PIB brasileiro (IBGE, 2012) e, segundo a Pesquisa Industrial Anual (PIA) em 2009, o setor empregou pelo menos 7,9 milhões de pessoas e gastou com pessoal R \$ 240,4 bilhões (IBGE, 2012). Dessa forma, trata-se de um setor fundamental para a geração de renda, riqueza e de empregos diretos e indiretos no país. Apesar da importância desse setor para a economia brasileira, há certa carência de estudos recentes sobre a demanda industrial por energia elétrica, sendo Castro e Rosental (2008) um dos mais recentes. Os demais estudos como os da EPE (2011) trataram apenas de previsões da demanda, sem considerar as elasticidades e seu comportamento conjuntural. Nesse sentido, a consecução da presente pesquisa visa contribuir com o debate acadêmico em pelo menos três vertentes. Primeiro, ao utilizar a mesma proposta metodológica dos autores supracitados, mas utilizando uma base de dados mais atual, busca-se verificar como as elasticidades preço, preço cruzado e renda da demanda de 
energia elétrica industrial no Brasil se alteraram ao longo dos últimos anos. Segundo, ao utilizar a metodologia Box-Jenkis para prever o crescimento da demanda de energia elétrica três passos à frente, como realizado em Siqueira e Hollanda (2005), Leite (2006) e EPE (2011), mas com dados mensais, diferentemente desses autores que realizaram previsões com base de dados anuais; a periodicidade mensal melhora a capacidade de captação das possíveis volatilidades do setor. Outra diferença em relação ao que se propôs realizar nesta pesquisa e os trabalhos citados anteriormente, diz respeito a proxy utilizada para representar o preço do bem substituto. No presente caso, utiliza-se o preço da commodity petróleo e derivados, enquanto os demais autores utilizavam o índice IPA-OG de combustíveis e lubrificantes.

Defronte aos pontos levantados, a proposta do artigo é responder ao seguinte questionamento: como reage a demanda de energia elétrica no Brasil frente à alteração na renda interna, no seu preço e no preço de um bem substituto, equal seria a previsão de crescimento da demanda para os próximos três períodos subsequentes (junho, julho e agosto de 2012).

Para responder a essa problemática delineia-se como objetivo geral analisar os determinantes da demanda de energia elétrica industrial no Brasil no período compreendido entre janeiro de 1990 e maio de 2012, e prever como irá se comportar essa demanda três passos à frente. Nesse contexto, como objetivos específicos, aponta-se, efetuar uma análise conjuntural para diagnosticar como se encontra o mercado de energia elétrica no Brasil, estimar os valores das elasticidades renda, preço cruzado e preço da demanda de energia elétrica industrial no país, bem como, realizar a previsão três passos à frente e estimar os erros de previsão, verificando a precisão da previsão.

Nesse aparato, com o intuito de atender aos objetivos apresentados, o presente artigo está dividido em cinco seções. A primeira contempla esta breve introdução, enquanto que a segunda seção traz uma breve contextualização do setor de energia elétrica industrial no Brasil. Já, a terceira e quarta seções apresentam, respectivamente a metodologia e os resultados obtidos. Por fim, a quinta e última seção contempla as conclusões finais.

\section{2) ANÁLISE CONJUNTURAL DO CONSUMO E DO CUSTO DA ENERGIA ELÉTRICA INDUSTRIAL NO BRASIL - 1990 A 2012}

O consumo de energia elétrica no setor industrial vem apresentando tendência positiva de crescimento, conforme observa-se na Figura 1. Em 
janeiro de 1990, o consumo era de 8.327.000 MWh, porém em maio de 2012 passou a ser de 15.467.000 MWh. Na comparação entre as duas datas isso significou um aumento de $85,75 \%$. A Figura 1 revela, além dessa evolução histórica no período de 1990 a 2012, a evolução do PIB no mesmo período. Ao se analisar a Figura 1 fica claro o acompanhamento entre o crescimento do consumo de energia elétrica e o crescimento do PIB.

Figura 1: Consumo de energia elétrica em relação ao PIB no setor industrial em MWh.

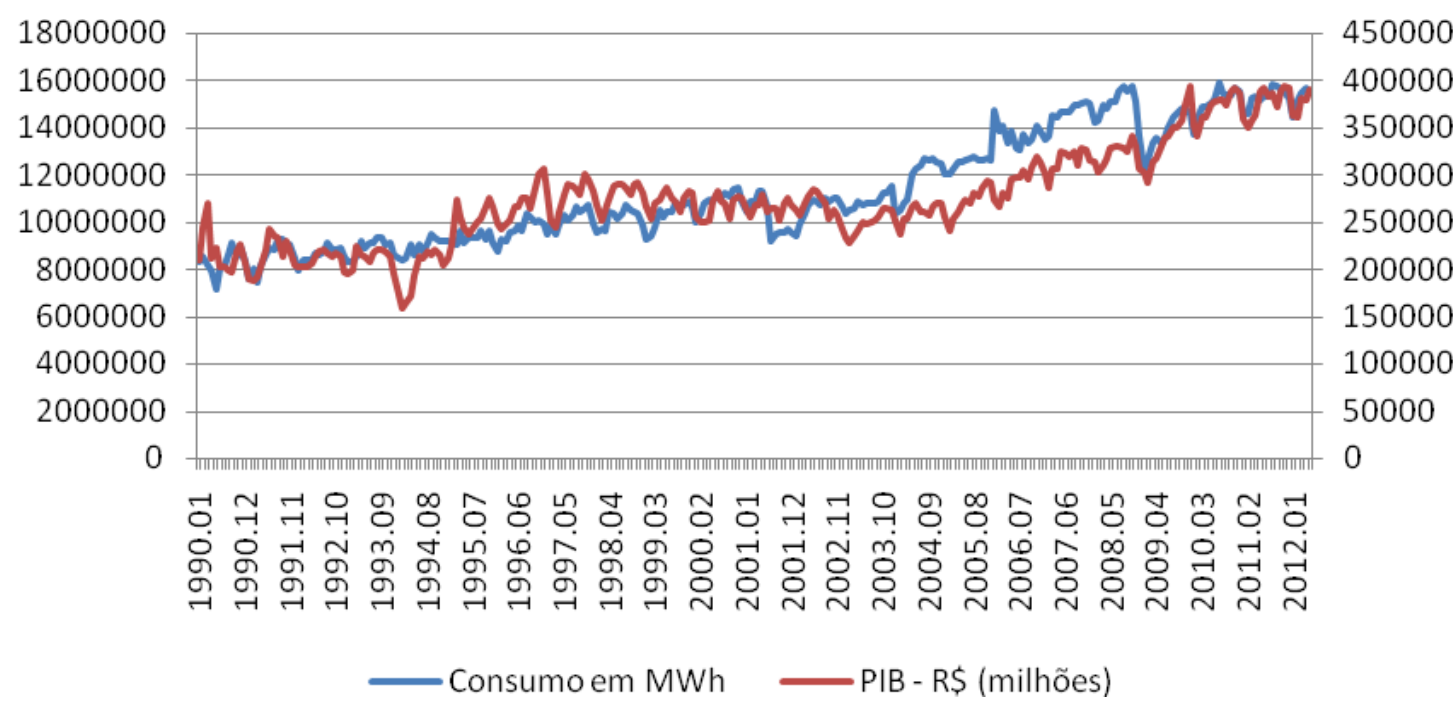

Fonte: Elaborada pelos autores com base nos dados disponíveis no IPEA (2012).

A taxa de crescimento da demanda de energia elétrica no setor industrial, representada através da regressão de um modelo log-linear do consumo de energia elétrica contra o tempo, foi de $0,2542 \%$ ao mês, para o período de janeiro de 1990 a maio de 2012. Por outro lado, na regressão de um modelo de tendência linear o coeficiente angular da variável de tempo apresentou sinal positivo. Ou seja, a demanda por energia elétrica no setor industrial possui uma tendência crescente. Entre janeiro de 1990 e maio de 2012 o consumo de energia elétrica no setor industrial aumentou, em média, 29.102,6 MWh ao mês.

A partir da implantação do plano real, em julho de 1994, a tarifa média por MWh da energia elétrica no setor industrial - em preços constantes de setembro de 2012 deflacionados pelo IGP-DI - aumentou 12,35\%. Enquanto em julho de 1994 esta tarifa era de R\$ 238,76 MWh, em maio de 2012 passou a custar R\$268,26 MWh, conforme pode ser observado na evolução 
histórica retratada na Figura 2. Na mesma figura, também se encontra o consumo em MWh.

Figura 2: Tarifa média em R \$ por MWh (deflator: IGP-DI (preços set. 2012 = 1)) da energia elétrica em relação com consumo em MWh.

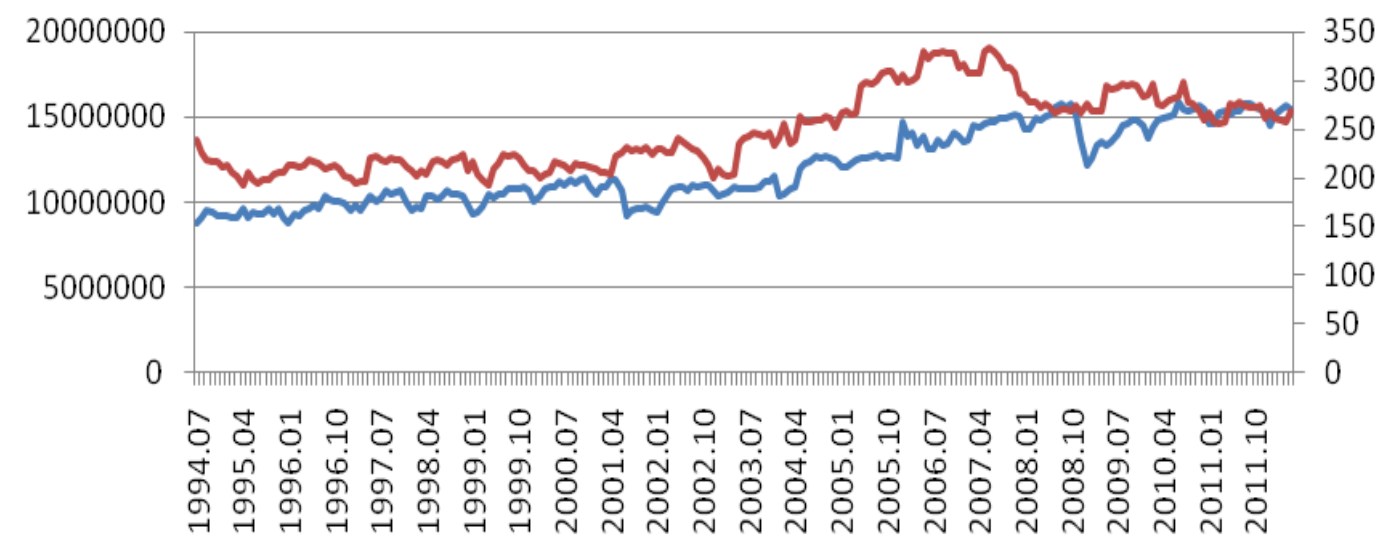

—Consumo em MWh —Tarifa média por MWh R\$

Fonte: Elaborada pelos autores com base nos dados disponíveis no IPEA (2012).

Na Figura 3 é mostrada a evolução do índice de preço do petróleo e derivados em comparação com a evolução do consumo de energia elétrica pelo setor industrial. A tendência aparenta ser similar para ambas as séries. De 2008 para 2009 ambas as séries tiveram uma queda brusca, facilmente justificada pela crise financeira de 2008 .

Figura 3: Índice de Preço do Petróleo e derivados em relação ao consumo em MWh no setor industrial.

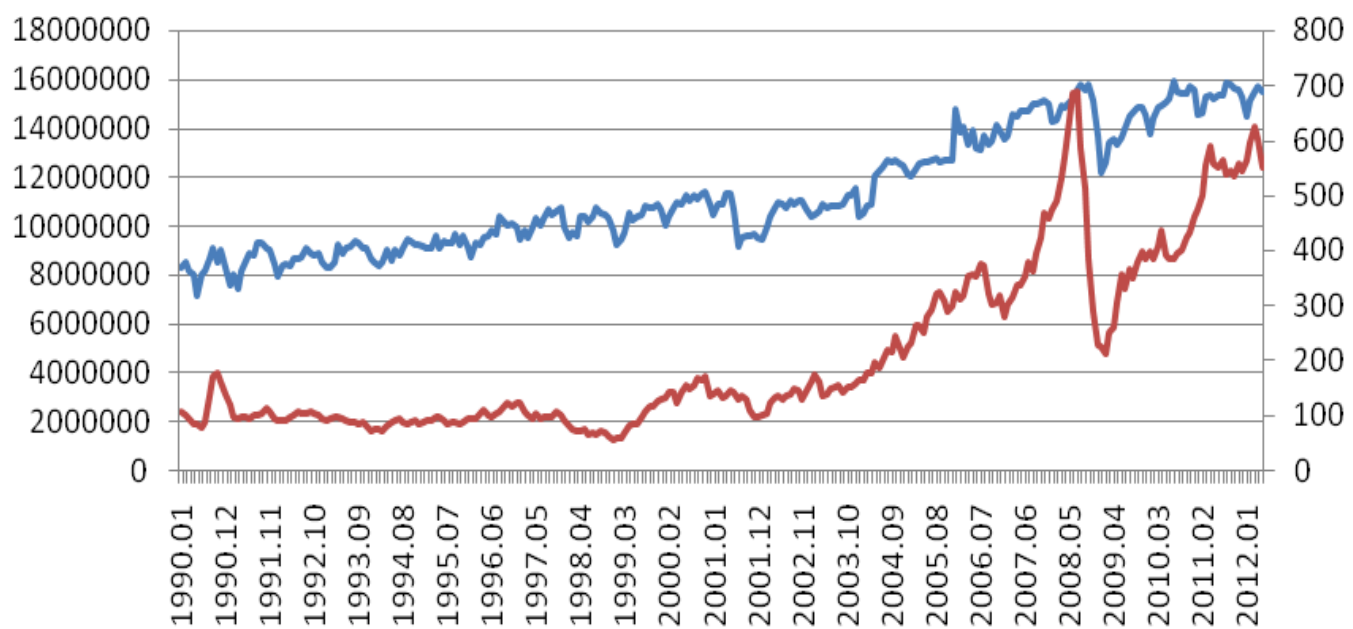

Consumo em MWh — Petróleo e derivados - preço - índice

Fonte: Elaborada pelos autores com base nos dados disponíveis no IPEA (2012) 
Segundo o Banco de Informações de Geração (BIG) da Agência Nacional de Energia Elétrica (ANEEL), o Brasil possui, atualmente, 2.697 empreendimentos geradores de energia elétrica, os quais respondem por $119.570 .598 \mathrm{~kW}$ de potência. Para os próximos anos, e também para um período mais longo (anos próximos a 2020), estão previstos aumentos de $48.450 .908 \mathrm{~kW}$ na capacidade de geração do país (ANEEL, 2012). A queda do consumo de energia elétrica pelo setor industrial no primeiro semestre de 2009, devido à crise financeira, contribuiu para evitar o aumento da tarifa de energia elétrica (INFRAESTRUTURA, 2011).

A Tabela 1 mostra a composição dos empreendimentos geradores de energia elétrica no Brasil. Nota-se que o maior número de empreendimentos está concentrado em termelétricas, porém os com maior potência são as usinas hidrelétricas.

O Brasil também possui oito usinas fotovoltaicas ${ }^{1}$ e um empreendimento de fonte maré de potência associada de $50 \mathrm{~kW}$. Esses empreendimentos são minoritários no Brasil por possuírem uma capacidade de geração muito baixa, além do seu custo de instalação ser elevado quando comparado a outras fontes, como a de hidroelétricas, onde o custo-benefício é muito maior no caso do Brasil. O Brasil também importa a maioria das placas fotovoltaicas, o que aumenta ainda mais o custo de instalação de tais usinas.

Tabela 1: Empreendimentos em operação².

\begin{tabular}{lcccc}
\hline Tipo & Quantidade & Potência outorgada $(\mathrm{kW})$ & Potência fiscalizada $(\mathrm{kW})$ & $\%$ \\
\hline CGH & 391 & 232.499 & 230.869 & 0,19 \\
EOL & 81 & 1.795 .028 & 1.718 .532 & 1,44 \\
PCH & 426 & 4.211 .277 & 4.137 .493 & 3,46 \\
UFV & 8 & 5.494 & 1.494 & 0 \\
UHE & 201 & 81.963 .837 & 79.048 .204 & 66,11 \\
UTE & 1.588 & 33.715 .651 & 32.427 .006 & 27,12 \\
UTN & 2 & 1.990 .000 & 2.007 .000 & 1,68 \\
Total & $\mathbf{2 . 6 9 7}$ & $\mathbf{1 2 3 . 0 1 3 . 7 8 6}$ & $\mathbf{1 1 9 . 5 7 0 . 5 9 8}$ & $\mathbf{1 0 0}$ \\
\hline
\end{tabular}

(Os valores de porcentagem são referentes a potência fiscalizada. A potência outorgada é igual a considerada no ato de outorga. A potência fiscalizada é igual a considerada a partir da operação comercial da primeira unidade geradora).

${ }^{1}$ São usinas que utilizam no processo de geração de energia módulos solares e, a energia gerada por estes módulos é chamada de energia solar fotovoltaica.

${ }_{2}^{2}$ CGH - Central Geradora Hidrelétrica; EOL - Central Geradora Eolielétrica; PCH - Pequena Central Hidrelétrica; UFV - Usina Fotovoltaica; UHE - Usina Hidrelétrica de Energia; UTE Usina Termelétrica de Energia; UTN - Usina Termonuclear; 
O Brasil possui apenas duas usinas termonucleares que respondem por $1,68 \%$ da produção nacional. Porém, quando comparada, por exemplo, com as centrais geradoras eolielétricas, que representam um total de 81 unidades no país e respondem por apenas $1,44 \%$ da produção nacional, revelam a sua alta capacidade de geração em pouquíssimas unidades produtoras.

A grande quantidade de termelétricas instaladas no país (1.588 unidades) mostra a facilidade com que o petróleo (mais especificamente o seu derivado óleo diesel) pode ser usado como substituto na geração de energia elétrica, a depender do preço de cada insumo, principalmente pelo setor industrial.

A Figura 4 mostra a composição da matriz de energia elétrica brasileira. As energias fósseis ainda são significativas no Brasil, como o petróleo e o gás natural, que juntos somam $16 \%$ da produção nacional. A importação de energia elétrica $(6,4 \%)$ supera a quantidade produzida por carvão mineral, a nuclear e a eólica juntas. Além de ser isoladamente maior que a produzida pelo petróleo.

Figura 4: Participação na matriz de energia elétrica em \% de cada fonte.

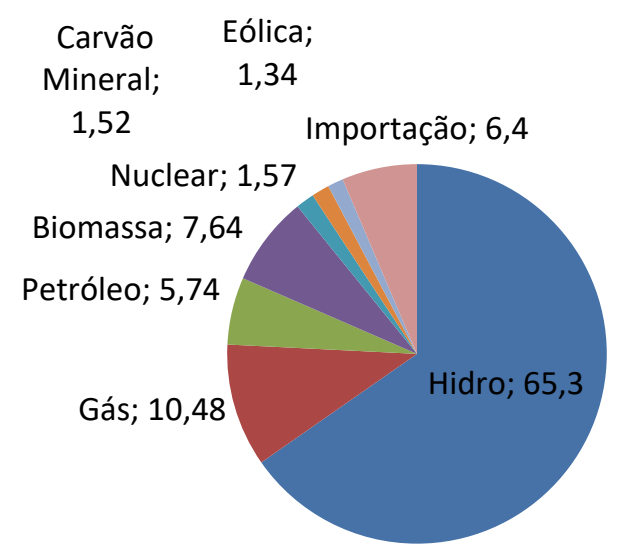

Fonte: ANEEL (2012).

Quando analisada a questão dos custos, se percebe que o custo financeiro da energia elétrica é outro fator que influencia muito na sua demanda. A energia elétrica brasileira é uma das mais caras do mundo. Há quatorze encargos que incidem sobre a indústria brasileira, que respondem no final por $17 \%$ da tarifa paga pela indústria e os tributos correspondem a 31,5\% (FIRJAN, 2011). A tarifa média de energia elétrica industrial no Brasil 
é de 329,00 reais por MWh, conforme mostra a Tabela 2. Trata-se da tarifa mais alta entre os países do BRIC. A média dos países do BRIC sem o Brasil é de140,7 R\$/MWh. A Rússia é o país do BRIC que possui a tarifa mais baixa ficando em 91,5R \$/MWh, ou seja, 259,56\% menor que a do Brasil. Todos os países do BRICS possuem uma tarifa, no mínimo,74,91\% menor que a do Brasil.

Tabela 2: Tarifas de energia elétrica industrial do BRIC - Brasil, Rússia, Índia e China (R\$/MWh).

\begin{tabular}{lc}
\hline \multicolumn{1}{c}{ Países } & $\begin{array}{c}\text { Tarifa média } \\
\text { (R } \$ / M W h)\end{array}$ \\
\hline Brasil & 329,00 \\
Índia & 188,10 \\
China & 142,40 \\
Rússia & 91,50 \\
Média de Rússia, Índia e China & 140,70 \\
\hline Fonte: FIRJAN (2011).
\end{tabular}

Essa discrepância se mostra pelo alto valor dos encargos e tributos existentes na energia brasileira. Essa tarifa elevada contribui para o aumento dos custos das indústrias brasileiras e na perda de competitividade no setor industrial.

\section{3) METODOLOGIA}

O presente estudo tem por referencial teórico a teoria da demanda neoclássica (VARIAN, 2006), em que a demanda de um bem normal pode ser representada como a seguir:

$$
D_{x}=f\left\{Y, P_{x}, P_{y}\right\}
$$

onde, $D_{x}$ é quantidade demandada do bem x, $Y$ é renda dos consumidores, $P_{x}$ é preço de venda do bem x e $P_{y}$ é preço de venda do bem substituto y.

A Equação 01 ilustra que a quantidade demandada de um bem normal " $x$ " é uma função linear da renda dos consumidores $(Y)$, do preço de comercialização do bem $\mathrm{x}(P x)$ e do preço de comercialização do bem substituto y $(P y)$.

No que se refere ao comportamento dos sinais esperados para os coeficientes dessas variáveis, tem-se o seguinte: sinal positivo para o coeficiente de $Y$, sendo que com um aumento da renda dos consumidores 
espera-se um aumento da demanda do bem $x$; sinal negativo para o coeficiente da variável $P x$, uma vez que aumentando o preço desse bem espera-se uma queda na sua demanda; e, sinal positivo para o coeficiente do preço de um bem substituto y, já que com o aumento do preço de Y, esperase o aumento da demanda do bem $x$.

Quanto aos dados utilizados na presente pesquisa, os mesmos foram obtidos junto ao Instituto de Pesquisa Econômica Aplicada (IPEA). A base de dados é mensal e compreende o período de janeiro de 1990 a maio de 2012 , sendo as seguintes variáveis utilizadas: Consumo de energia elétrica do setor industrial em $\mathrm{MWh}^{3}$; Tarifa média de energia elétrica industrial por $\mathrm{MWh}^{4}$;Proxy para o preço de um bem substituto, usando para isso o índice de preços internacionais de commodities de petróleo e derivados ${ }^{5}$; e, Produto interno bruto (PIB) ${ }^{6}$.

\section{1) Modelo Econométrico}

O modelo utilizado é do tipo duplo-log. Um aspecto muito atraente deste modelo, que o tornou apreciável nos trabalhos aplicados, é que o coeficiente angular dessa forma funcional mede a elasticidade (variação percentual) da variável dependente em relação a variável independente.

O modelo tem a seguinte especificação ${ }^{7}$ :

$$
\ln C_{t}=\alpha-\beta_{1} \ln P_{t}+\beta_{2} \ln Y_{t}+\beta_{3} \ln P B S_{t}+u_{t}
$$

em que,

$\ln C$ : logaritmo natural da quantidade consumida de energia elétrica;

$\ln P$ : logaritmo natural da tarifa média da energia elétrica;

$\ln Y$ : logaritmo natural do PIB;

$\ln P B S$ : logaritmo natural do preço de um bem substituto;

$u_{t}$ : termo de erro;

$\alpha$ : intercepto do modelo;

$\beta_{1}$ : Coeficiente que revela a elasticidade preço da demanda;

3 Os dados brutos do IPEA encontravam-se em GWh, para transformar para MWh foi multiplicado por 1000. Fonte: Eletrobrás.

${ }^{4}$ Deflator: IGP-DI (preços set. $2012=1$ ). Fonte: Eletrobrás. Unidade R\$.

${ }^{5}$ Fonte: IPEA. Índice (jan. $\left.2002=100\right)$.

${ }^{6}$ Deflator: IGP-DI (preços set. 2012 = 1). Fonte: Banco Central do Brasil, Boletim, Seção Atividade Econômica (BCB Boletim/Ativ. Ec.). Unidade: R \$ (milhões).

7 Para o cálculo da regressão e de todos os testes econométricos será utilizado o software STATA. 
$\beta_{2}$ : Coeficiente que revela a elasticidade preço cruzada da demanda;

$\beta_{3}$ : Coeficiente que revela a elasticidade renda da demanda;

$\mathrm{Na}$ equação 02 tem-se o modelo $\log$-log, onde $\alpha$ é o intercepto e os coeficientes $\beta_{i}$ são chamados de coeficientes parciais de regressão. Estes coeficientes medem os impactos das mudanças de uma variável independente em relação à variável dependente, mantendo todas as demais variáveis do modelo constantes.

\section{2) Previsão Utilizando a Metodologia Box-Jenkins}

A previsão pela metodologia ARIMA é conhecida popularmente pelo métodoBox-Jenkins. O enfoque desses métodos não está na construção de modelos de equações simultâneas ou uniequacionais, mas sim na análise probabilística, ou estocástica, nas propriedades da própria séria temporal, com a filosofia data driven. Nessa metodologia o $Y_{t}$ será explicado por seus valores passados, ou defasados dos termos do erro estocástico (GUJARATI, D. N.; PORTER, D. C., 2011).

Por essas especificidades tais modelos são considerados ateóricos, pois não são derivados de nenhuma teoria econômica. No processo autorregressivo integrado de médias móveis (ARIMA) $(p, d, q)$, identifica-se o número de termos de autorregressivos $(p)$, o número de vezes que a série deve ser diferenciada para que se torne estacionária $(d)$ e o número de termos de médias móveis $(q)$ (BUENO, 2008). Analiticamente, um modelo ARIMA $(p, d, q)$ pode ser especificado da seguinte forma:

$$
Y_{t}=\delta+\sum_{1}^{n} \alpha_{n} Y_{t-n}+u_{t}+\sum_{1}^{q} \gamma_{q} u_{t-q}
$$

em que,

$Y_{t}$ : É a previsão do consumo de energia elétrica;

$\delta$ : É o intercepto;

$\sum_{1}^{n} \alpha_{n} Y_{t-n}$ : É o somatório variando de 1 até $n$ dos coeficientes $\alpha_{n}$ do operador de diferenças AR $\left(Y_{t-n}\right)$;

$u_{t}$ : Termo de erro;

$\sum_{1}^{q} \gamma_{q} u_{t-q}$ : É o somatório variando de 1 até $q$ dos coeficientes $\beta_{q}$ do operador de diferenças MA $\left(u_{t-q}\right)$. 


\section{4) RESULTADOS E DISCUSSÕES}

Num primeiro momento, a presente seção contempla os resultados da estimação do modelo de longo e de curto prazo para o consumo de energia elétrica, enquanto que num segundo momento apresenta o consumo de energia previsto através da metodologia Box-Jenkins para períodos selecionados.

\section{1) Estimação das Relações de Longo e de Curto Prazo}

Através dos testes de DickeyFuller, Phillips Perron, KPSS e NG-Perron as séries utilizadas se mostraram não estacionárias em nível. Porém, em primeira diferença todas as séries se tornaram estacionárias (Tabela 4).

Tabela 4: Testes de Estacionariedade de DFA, Phillips-Perron, KPSS e NG-PERRON.

\begin{tabular}{|c|c|c|c|c|c|}
\hline Variável & DFA* & PP* & KPSS $^{* * *}$ & $\begin{array}{l}\text { NG- } \\
\text { Perron } \\
M Z a M Z a \\
* * *\end{array}$ & $\begin{array}{l}\text { NG- } \\
\text { Perron } \\
M Z t M Z t \\
* * *\end{array}$ \\
\hline $\operatorname{lnClags}(1)$ & $-1,201$ & $-1,175$ & 12,499 & -0.061 & -0.033 \\
\hline lnPlags(1) & $-1,979$ & $-1,962$ & 4,33 & -6.697 & -1.829 \\
\hline lnYlags(4) & $-0,713$ & $-1,6$ & 4,12 & 0.751 & 0.446 \\
\hline lnPBSlags(2) & $-1,063$ & $-0,749$ & 7,49 & -1.289 & -0.490 \\
\hline $\operatorname{lnCdiflags(0)}$ & $-17,752$ & $-17,961$ & 0,0125 & -172.870 & -9.287 \\
\hline lnPdiflags(0) & $-14,734$ & $-14,732$ & 0,0966 & -139.585 & -8.354 \\
\hline lnYdiflags(4) & $-9,025$ & $-15,178$ & 0,0323 & -10.279 & -2.236 \\
\hline lnPBSdiflags(1) & $-9,389$ & $-11,62$ & 0,107 & -78.903 & -6.275 \\
\hline
\end{tabular}

Fonte: Resultados da pesquisa.* Valor crítico a 5\% de significância é de $-2,879 . * *$ Valor crítico a $5 \%$ de significância é de 0,463 . *** Teste de NG-Perron, valor crítico ao nível de significância de $5 \%$ igual a $-8,10$ para $M Z_{a}$ e $-1,98$ para $M Z_{t}$, a $1 \%$ são respectivamente, $-13,80$ e $-2,58$.

Com base na Tabela 5 é possível notar que há pelo menos um vetor de cointegração, uma vez que a estatística traço calculada de 24,9752 ao nível de significância de $5 \%$ é menor que o valor crítico de 29,68 , levando a não rejeição de $H_{0}$ (existe pelo menos 1 vetor de cointegração). Ou seja, existe uma relação de longo prazo entre as séries utilizadas no modelo. 
Tabela 5: Teste de Co-integração de Johansen.

\begin{tabular}{cccccc}
\hline $\begin{array}{c}\text { Vetores de } \\
\text { cointegração }\end{array}$ & $\begin{array}{c}\text { Estatística } \\
\text { Traço }\end{array}$ & $\begin{array}{c}\text { Valor } \\
\text { crítico } \\
\mathbf{5 \%}\end{array}$ & $\begin{array}{c}\text { Vetores de } \\
\text { cointegração }\end{array}$ & $\begin{array}{c}\text { Estatística } \\
\text { autovalor }\end{array}$ & $\begin{array}{c}\text { Valor } \\
\text { Crítico } \\
\mathbf{5 \%}\end{array}$ \\
\hline Nenhum & 55,0806 & 47,21 & $\mathrm{r}=0$ & 30,1054 & 27,07 \\
Pelo menos 1 & $24,9752^{*}$ & 29,68 & $\mathrm{r}=1$ & $18,8946^{*}$ & 20,97 \\
Pelo menos 2 & 6,0806 & 15,41 & $\mathrm{r}=2$ & 4,6941 & 14,07 \\
Pelo menos 3 & 1,3864 & 3,76 & $\mathrm{r}=3$ & 1,3864 & 3,76 \\
\hline
\end{tabular}

Fonte: Elaborado pelos autores com base nos resultados da pesquisa.

Os resultados do modelo mostram que todos os parâmetros são significativos a 1\%, conforme se pode verificar na Tabela 6 através do valor $p$, com exceção da variável preço. No entanto, dada a importância desta variável para a análise em questão, ou seja, a elasticidade preço, mantém-se esta variável no modelo. Os sinais encontrados foram condizentes com a teoria. Negativo para Preço e positivo para a Renda e Preço do Bem Substituto. Ou seja, à medida que o preço da energia elétrica aumenta a sua demanda diminui. Por outro lado, à medida que o preço do Petróleo e a Renda aumentam, a demanda por energia elétrica também aumenta.

Com relação aos valores dos parâmetros, pode-se dizer que, dado um aumento de $1 \%$ no Preço da energia elétrica, o seu Consumo irá diminuir, em média, 0,006\%, tudo o mais permanecendo constante. Ao passo que, se ocorrer um aumento de $1 \%$ no preço do Petróleo, o Consumo de energia elétrica irá aumentar, em média, 0,1868\%. Enquanto que um aumento de $1 \%$ na Renda aumentará o Consumo de energia elétrica, em média, em 0,4490\%, ceterisparibus.

Tabela 6: Estimação das relações de longo prazo.

\begin{tabular}{lccc}
\hline \multicolumn{1}{c}{ Variável } & Coeficiente $^{*}$ & Teste $\boldsymbol{t}$ & Valor $\boldsymbol{p}$ \\
\hline Constante & $-9,6985$ & --- & --- \\
$\ln \mathrm{P}$ & $-0,0066(0,0043)$ & 1.54 & 0.124 \\
$\ln \mathrm{Y}$ & $0,4490(0,0894)$ & -5.02 & 0,00 \\
$\operatorname{lnPBS}$ & $0,1868(0,0225)$ & -8.27 & 0,00 \\
\hline * Os valores entre parênteses indicam desvios-padrão. \\
Fonte: Elaborado pelos autores.
\end{tabular}

A elasticidade renda da demanda se mostrou significativa e estatisticamente maior que zero $(0,4490)$. A elasticidade preço da demanda se 
mostrou inelástica $(-0,0066)$, o que significa que a quantidade demandada é relativamente pouco sensível às variações do preço. Por outro lado, a elasticidade-preço cruzada da demanda foi de $(0,1868)$ o que mostra que o petróleo é um bem substituto para a energia elétrica usada no setor industrial, o que significa que quando o preço do petróleo varia positivamente, tudo o mais permanecendo constante, tende a aumentar a demanda por energia elétrica.

Na Tabela 7 faz-se uma comparação entre os valores encontrados por outros autores que analisaram a demanda por energia elétrica em diferentes locais, períodos e métodos. A primeira diferença que se percebe é que todos os parâmetros apresentaram valores menores do que os trabalhos que usaram dados anuais. Os sinais encontrados foram condizentes com os demais trabalhos, negativo para a tarifa de energia elétrica e positivo para as demais variáveis, variando no caso da constante que em alguns teve sinal positivo e em outros negativos.

Tabela 7: Comparação dos resultados encontrados com os de outros autores.

\begin{tabular}{|c|c|c|c|c|c|c|}
\hline $\begin{array}{l}\text { Autores/Período de } \\
\text { Análise }\end{array}$ & $\begin{array}{l}\text { Método/ } \\
\text { Local }\end{array}$ & Constante & Tarifa & $\begin{array}{l}\text { Preço de } \\
\text { Máquinas }\end{array}$ & Renda & $\begin{array}{l}\text { Preço de } \\
\text { Bem } \\
\text { Substituto }\end{array}$ \\
\hline $\begin{array}{l}\text { Siqueira \&Hollanda } \\
2005)^{* / 1} 1970 \text { a } 2000\end{array}$ & $\begin{array}{l}\text { MQO/ } \\
\text { Nordeste }\end{array}$ & $-5,32$ & -0.87 & -0.55 & 1,58 & 0.33 \\
\hline $\begin{array}{l}\text { Siqueira \&Hollanda } \\
(2005)^{* / 1} 1970 \text { a } 2000\end{array}$ & $\begin{array}{l}\text { MQ2E/ } \\
\text { Nordeste }\end{array}$ & $-6,38$ & -0.57 & -0.65 & 1,67 & 0.26 \\
\hline $\begin{array}{l}\text { Siqueira \&Hollanda } \\
(2005)^{* / 1} 1970 \text { a } 2000\end{array}$ & $\begin{array}{l}\text { Johansen/ } \\
\text { Nordeste }\end{array}$ & -529 & -0.44 & -0.85 & 0.99 & 0.08 \\
\hline $\begin{array}{l}\text { IrffiI. et al. (2009)*/ } \\
1970 \text { a } 2003\end{array}$ & $\begin{array}{l}\text { DOLS/ } \\
\text { Nordeste }\end{array}$ & 8,03 & $-3,71$ & 0,96 & 1,21 & 0,51 \\
\hline $\begin{array}{l}\text { Schmidt \& Lima } \\
(2004)^{* / 1} 1969 \text { a } 1999\end{array}$ & $\begin{array}{c}\text { Johansen/ } \\
\text { Brasil }\end{array}$ & 2,36 & $-0,55$ & $-0,46$ & 1,92 & 0,02 \\
\hline $\begin{array}{l}\text { Mattos (2005)/ } 1974 \text { a } \\
2002\end{array}$ & $\begin{array}{l}\text { Johansen/ } \\
\text { Brasil }\end{array}$ & 6,8 & $-0,49$ & - & 1,59 & - \\
\hline
\end{tabular}

O período mais recente analisado foi até 2003 , no trabalho de Irffi et al. (2009), enquanto que o presente trabalho analisou dados até maio de 2012. Percebe-se que os resultados encontrados nesse trabalho para a elasticidadepreço da demanda são menores $(-0,006)$, da mesma forma que para a elasticidade-renda da demanda $(0,45)$. 
Schmidt e Lima (2004) e Mattos (2005) analisaram os dados para o Brasil como um todo, os demais autores analisaram apenas a região nordeste do Brasil. Os resultados foram muito semelhantes para o Brasil e para a Região Nordeste, indicando ser relativamente homogênea a demanda no Brasil. O método mais usado para estimação foi o Johansen, seguido por MQO e outros métodos menos comuns como MQ2E e DOLS.

Já, na Tabela 8,apresentam-se os resultados estimados do modelo de curto prazo, juntamente com o Mecanismo de Correção de Erros.

Os sinais encontrados para as variáveis estão de acordo com a teoria econômica. $\mathrm{O}$ sinal positivo para a renda indica que a medida que a renda aumenta a demanda pelo bem também aumenta. Um sinal positivo para o preço do bem substituto, ou seja, a medida que aumenta o preço do bem substituto aumenta também a demanda pelo bem analisado, neste caso, um aumento do preço do petróleo leva a um aumento na demanda por energia elétrica no setor industrial.

Tabela 8: Modelo de curto prazo por MCE.

\begin{tabular}{lcccc}
\hline & Coeficiente & Erro Padrão & Teste $\boldsymbol{t}$ & Valor $\boldsymbol{p}$ \\
\hline Constante & 0,0013 & 0,0022 & 0,61 & 0,541 \\
$\Delta \ln C_{\mathrm{t}-1}$ & $-0,1475$ & 0,0648 & $-2,27$ & 0,023 \\
$\Delta \ln \mathrm{P}_{\mathrm{t}-1}$ & 0,0046 & 0,0039 & 1,17 & 0,241 \\
$\Delta \ln \mathrm{Y}_{\mathrm{t}-1}$ & 0,1350 & 0,0476 & 2,84 & 0,005 \\
$\Delta \ln P B S_{\mathrm{t}-1}$ & 0,1116 & 0,0275 & 4,06 & 0,00 \\
$\varepsilon_{\mathrm{t}-1}$ & $-0,0799$ & 0,0412 & $-1,94$ & 0,052 \\
\hline
\end{tabular}

Fonte: elaborado pelos autores com base nos resultados da pesquisa.

Apenas o preço do bem em questão (tarifa da energia elétrica do setor industrial) que apresentou um sinal diferente do esperado pela teoria econômica. O sinal esperado seria negativo para o preço do bem, pois a medida que seu preço aumenta sua demanda se retrai, mas o sinal encontrado foi positivo, os resultados mostram que no curto prazo a medida que o preço aumenta o consumo também aumenta, porém esse mesmo parâmetro também se mostrou não significativo, ou seja, estatisticamente é igual a zero, somente ao nível de significância $(\alpha)$ de $25 \%$ o parâmetro seria significativo, como tal nível de significância é muito elevado não o aceitamos, ou seja, o valor do parâmetro é estatisticamente igual a zero. A constante também se mostrou não significativa, todos os demais parâmetros se mostraram significativos a $5 \%$ de significância. 
A não significância do $\ln P$ no curto prazo reflete um atraso temporal com que a demanda por energia elétrica responde as oscilações no preço de sua tarifa. Os resultados serão percebidos e absorvidos com certa defasagem temporal pelo sistema econômico, ou seja, políticas de redução ou aumento de preço da tarifa de energia elétrica não surtem efeito imediato na demanda industrial por energia elétrica.

O coeficiente de ajustamento $\left(\varepsilon_{t-1}\right)$ se mostrou significativo a $10 \%$ de significância e uma velocidade de ajustamento lenta, da ordem de $7 \%$ a cada período. Sendo o coeficiente negativo (-0,0799349), temos um desequilíbrio transitório (curto prazo) e sua velocidade de correção, rumo ao equilíbrio de longo prazo, é lenta. Assim, conclui-se que desequilíbrios de curto prazo da demanda de energia elétrica no setor industrial, devido a variações do preço, da renda e do preço do bem substituto (petróleo) são reduzidos lentamente. Este coeficiente de ajustamento indica qual a proporção do desequilíbrio de curto prazo do consumo de energia elétrica que é corrigido no mês seguinte. $\mathrm{O}$ valor encontrado de $7 \%$ é a diferença entre o valor realizado e o valor de longo prazo, corrigido a cada período.

Conforme a Figura 5, a análise da função de resposta ao impulso mostra que variações não antecipadas na renda e no preço do bem substituto (petróleo) têm impactos positivos na demanda de energia elétrica industrial. No caso de impulsos pelo lado da renda, esta demanda aumenta positivamente no primeiro mês, caindo no segundo, mas ainda com uma relação positiva. A mesma volta a crescer no terceiro mês, estabilizando-se a partir do quarto. No caso do $\ln P B S$, percebe-se que sua influência aumenta com o passar do tempo (nos três primeiros meses), após o terceiro mês seus efeitos se tornam estáveis.

Os efeitos de impulsos nos preços praticamente não são sentidos. Apresentam uma estabilização desde o início em torno de zero, semelhante ao parâmetro de curto prazo que foi estatisticamente igual a zero. Rapidamente os choques no preço são absorvidos pelo sistema. Este resultado vai em direção ao fato de a demanda por energia elétrica industrial ser altamente inelástica. 
Figura 5: Função de impulso resposta sobre a demanda de energia elétrica industrial.
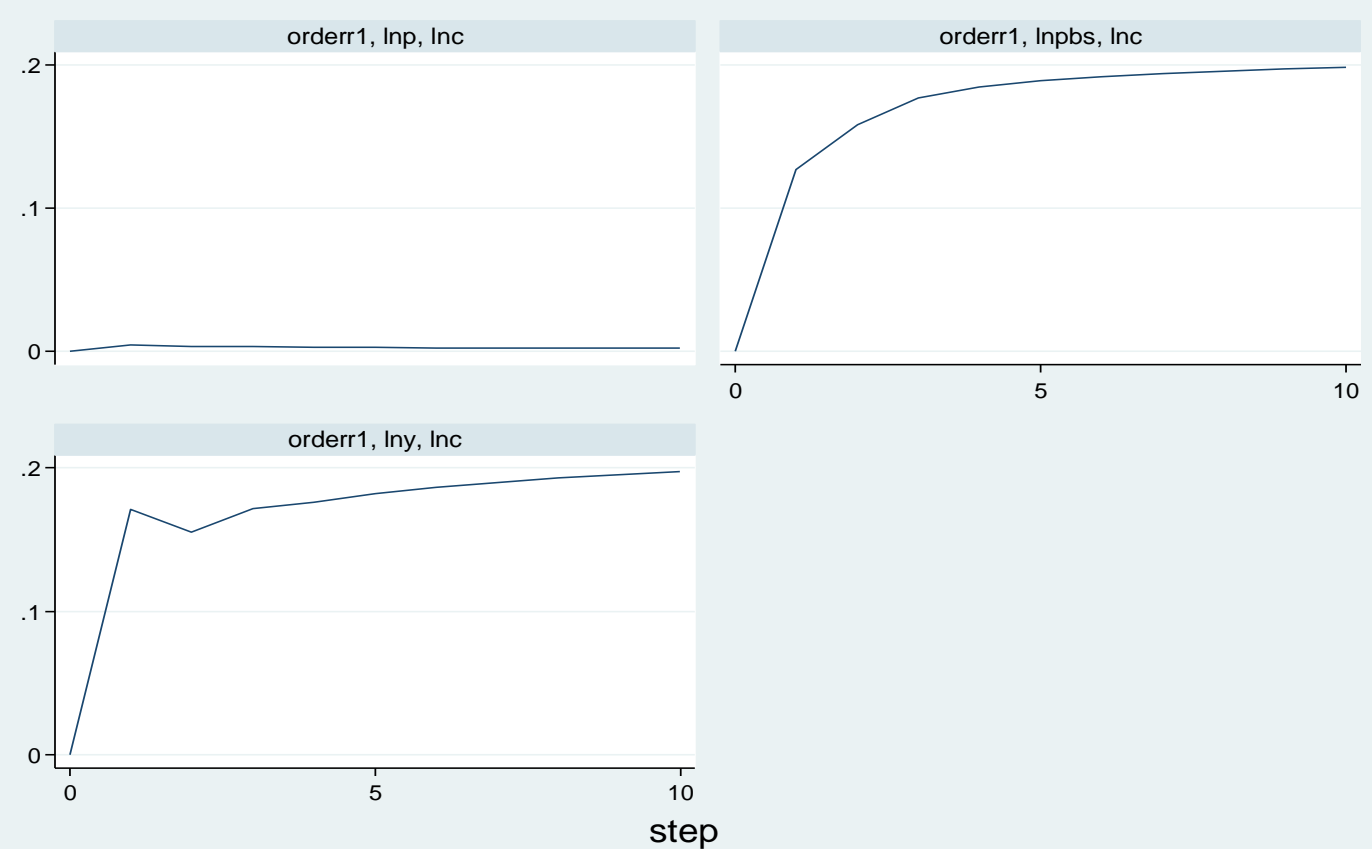

Fonte: Elaborado pelos autores com base nos resultados da pesquisa.

\section{2) Previsão Utilizando a Metodologia Box-Jenkins}

$\mathrm{Na}$ aplicação da metodologia Box-Jenkins para a previsão de determinada série temporal, é importante seguir três estágios principais. O primeiro contempla o processo de identificação dos possíveis modelos e de seus parâmetros. No segundo passo é elaborada a estimação do modelo, e por fim, efetuam-se os testes de adequação do modelo, aos quais se segue a aplicação do modelo para a previsão ${ }^{8}$.

Inicialmente, apresenta-se a Tabela 9, a qual traça um comparativo entre os períodos, locais, setores e métodos usados por outros autores que também realizaram previsões para a demanda de energia elétrica. Verifica-se que os autores, geralmente, usam ou a metodologia VAR ou a ARIMA, ambas as metodologias possuem bom poder de previsão. $\mathrm{Na}$ metodologia VAR, assim como nas equações simultâneas, deve-se considerar muitas variáveis endógenas em conjunto, não havendo, normalmente, variáveis exógenas no modelo. Levando em conta essas informações a opção neste trabalho foi pela metodologia ARIMA.

${ }^{8}$ Ver Hamilton (1994) e Enders (2010). 
Através da Tabela 9 percebe-se que todos os trabalhos de previsão foram para a demanda industrial, com exceção de Leite (2006) que fez a previsão da demanda para o país como um todo. Dos modelos utilizados, apenas Schmidt e Lima (2004) a fizeram para todo o Brasil, os demais autores se detiveram na Região Nordeste (IrffiI. et al. (2009), Siqueira e Hollanda (2005). O método usado pelos autores se concentra na metodologia VAR/VEC, sendo que apenas Leite (2006) usou a metodologia ARIMA para previsão. No entanto, esse último autor utilizou os dados de forma agregada para todo o Brasil e fez a previsão até o ano de 2012.

Conforme já analisado, a série $\ln C$ é estacionária em primeira diferença conforme os testes de DFA, PP, KPSS e NG-Perron já comentados. Para a identificação de $p$ e $q$, Gujaratie Porter (2011) e Bueno (2008) recomendam analisar a função de autocorrelação e de autocorrelação parcial da variável. $\mathrm{Na}$ Figura 6estão representadas a função de autocorrelação (FAC)e de autocorrelação parcial (FACP) da variável lnCdif. Segundo Bueno (2008), a escolha do modelo de previsão dá-se, principalmente, pela análise das funções de autocorrelação e de autocorrelação parcial. Assim, o padrão autoregressivo (AR) do modelo é determinado quando se verifica que a FACP é truncada na defasagem $p$ e a FAC apresenta uma queda exponencial da autocorrelação. Por outro lado, tratando-se de um modelo de médias móveis (MA), a FAC deve ser truncada na defasagem $q$ e a FACP deve apresentar um padrão de queda exponencial.

Tabela 9: Comparativo de previsões para demanda industrial de energia elétrica

\begin{tabular}{lccccc}
\hline Autores & $\begin{array}{c}\text { Período de } \\
\text { Análise }\end{array}$ & Previsão & $\begin{array}{c}\text { Método de } \\
\text { previsão }\end{array}$ & Demanda & Local \\
\hline $\begin{array}{l}\text { Siqueira e Hollanda } \\
(2005)\end{array}$ & 1970 a 2000 & $2004-2010$ & VAR/VEC & Industrial & Nordeste \\
$\begin{array}{l}\text { Schmidt e Lima } \\
(2004)\end{array}$ & 1969 a 1999 & $2001-2005$ & VAR/MCEV & Industrial & Brasil \\
IrffiI. et al. (2009) & 1970 a 2003 & $2004-2006$ & MCE/VEC & Industrial & Nordeste \\
Leite (2006) & 1966 a 2005 & $2006-2015$ & ARIMA & Nacional & Brasil \\
\hline onte: elaborado pelos autores. & & & & &
\end{tabular}

Assim, através da análise da Figura 6 nota-se que a função de autocorrelação é truncada na quarta e na décima segunda defasagem, tendo indícios de modelo degenerado MA $(4,12)$. Do mesmo modo, a função de autocorrelação parcial é truncada nestas mesmas defasagens, apresentando indícios de um modelo AR $(4,12)$. No entanto, como tanto a FAC quanto a 
FACP encontram-se truncadas nas defasagens especificadas, tal modelo pode ser especificado como um modelo degenerado $\operatorname{AR}(4,12)$ MA $(4,12)$.

Figura 6: Função de Autocorrelação (esquerda)e Função de Autocorrelação Parcial (direita) da variável lnCdif.
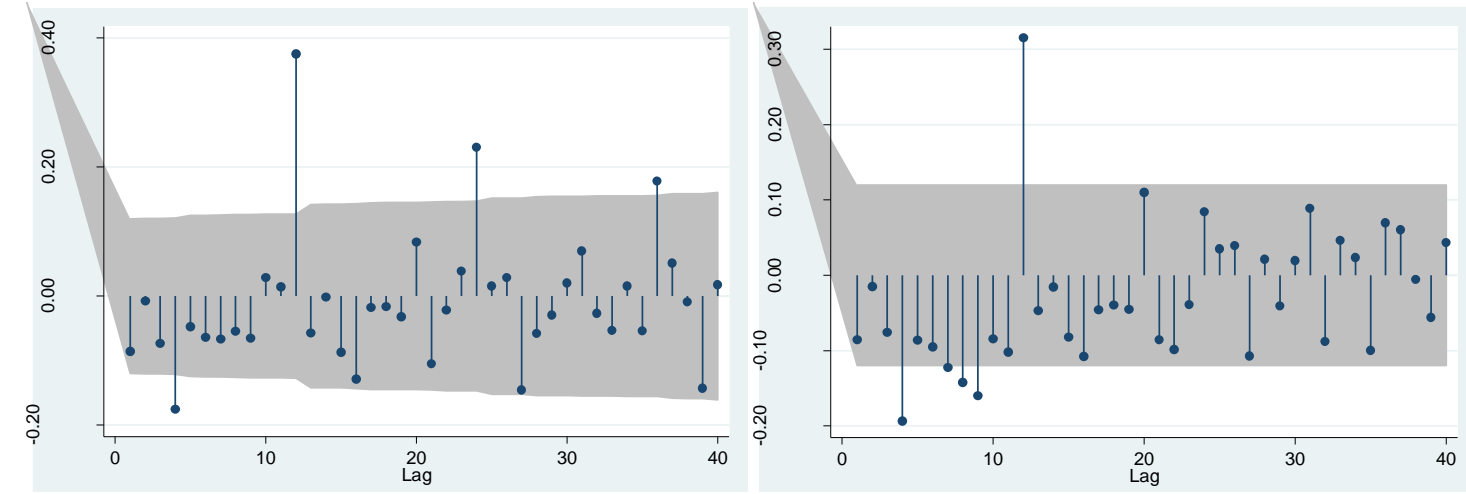

Fonte: Elaborado pelos autores com base nos resultados da pesquisa.

Juntamente com o processo de identificação do modelo, encontra-se o processo de decisão sobre qual modelo melhor se adéqua aos dados. Para isso,utilizaram-se os critérios de informação de AIC e BIC representados na Tabela 10.

Tabela 10: AIC e BIC para os modelos candidatos a previsão.

\begin{tabular}{ccc}
\hline & AIC & BIC \\
\hline MA(4,12) & $-1025,65$ & $-1011,285$ \\
AR $(4,12)$ & $-1040,59$ & $-1026,229$ \\
AR $(4,12)$ & $-1052,761$ & $-1031,215$ \\
MA (4,12) & & \\
\hline Fonte: Elaborada pelos autores com base nos resultados da pesquisa.
\end{tabular}

Através das estatísticas presentes na Tabela 10, infere-se que, segundo Bueno (2008), quanto mais parâmetros são estimados em um mesmo período da amostra, menor será o erro estimado, no entanto, há também uma penalização pelo acréscimo destes estimadores ${ }^{9}$. Assim, deseja-se o modelo com o menor valor para as estatísticas de AIC e BIC. Nesse caso, o modelo com menor valor para os critérios de informação encontra-se no modelo AR $(4,12)$ MA $(4,12)$.

Desse modo estima-se o seguinte modelo:

\footnotetext{
${ }^{9}$ Para mais detalhes sobre os critérios de informação ver Bueno (2008, p. 46-48).
} 


$$
\ln C_{t}^{*}=\delta+\alpha_{4} \ln C_{t-4}^{*}+\alpha_{12} \ln C_{t-12}^{*}+\gamma_{4} u_{t-4}+\gamma_{12} u_{t-12}+u_{t}
$$

em que, $\ln C^{*}$ representa a primeira diferença do logaritmo do consumo de energia elétrica $(C)$.

Em seguida, a Tabela 11 contempla os valores para os parâmetros do modelo AR $(4,12)$ MA $(4,12)$ escolhido com base na análise da FAC e da FACP e, principalmente, com base no teste e avaliação dos possíveis modelos a serem estimados. Com exceção da constante e do parâmetro $\alpha_{4}$, todos os demais parâmetros se mostraram significativos, no entanto, os parâmetros não significativos foram mantidos no modelo, dado o bom grau de ajustamento proporcionado por este modelo.

No processo de verificação da adequação do modelo, parte-se para a análise dos resíduos provenientes do modelo $\operatorname{AR}(4,12)$ MA $(4,12)$. Neste passo, efetua-se a análise da autocorrelação dos resíduos. A Figura 7 mostra aautocorrelação dos resíduos. Portanto, têm-se indícios estatísticos que os resíduos do modelo têm distribuição normal. Adicionalmente, usa-se a estatística Box-Pierre $Q^{10}$ sobre os resíduos estimados para confirmar e reforçar os resultados. Assim, a soma dos quadrados dos resíduos das 30 autocorrelações dos resíduos, demonstrada pela estatística $Q$ é estatisticamente significativa (valor $p$ de 0,1853 ) confirmando que o termo de erro é ruído branco. Ou seja, tanto a FAC quanto a estatística Box-Pierre Q indicam que os resíduos estimados são puramente aleatórios, não havendo necessidade de se procurar por outro modelo ARIMA (GUJARATI \& PORTER, 2011).

Tabela 11: Parâmetros estimados para o modelo AR $(4,12)$ MA $(4,12)$.

\begin{tabular}{ccccc}
\hline & Coeficiente & Erro Padrão & Teste $\boldsymbol{t}$ & Valor $\boldsymbol{p}$ \\
\hline Constante & 0,00205 & 0,00411 & 0,50 & 0,617 \\
$\alpha_{4}$ & 0,02718 & 0,02767 & 0,98 & 0,326 \\
$\alpha_{12}$ & 0,95436 & 0,02800 & 34,08 & 0,000 \\
$\gamma_{4}$ & $-0,13425$ & 0,06485 & $-2,07$ & 0,038 \\
$\gamma_{12}$ & $-0,77283$ & 0,07821 & $-9,88$ & 0,000 \\
\hline
\end{tabular}

Fonte: Elaborado pelos autores com base nos resultados da estimação.

\footnotetext{
${ }^{10} \mathrm{~A}$ estatística $\mathrm{Q}$ é frequentemente utilizada como um teste para verificar se uma série temporal é um ruído branco. $Q=n \sum_{k=1}^{m} \rho_{k}^{2}$, em que $\mathrm{n}=$ tamanho da amostra, $\rho_{k}^{2}=$ coeficientes de correlação amostral e m o tamanho da defasagem. Com $\mathrm{H}_{0}: \rho_{k i}=0$ e $\mathrm{H}_{1}: \rho_{k i} \neq 0$.
} 
Figura 7: Função de Autocorrelação dos resíduos do modelo $\operatorname{AR}(4,12) \mathrm{MA}(4,12)$.

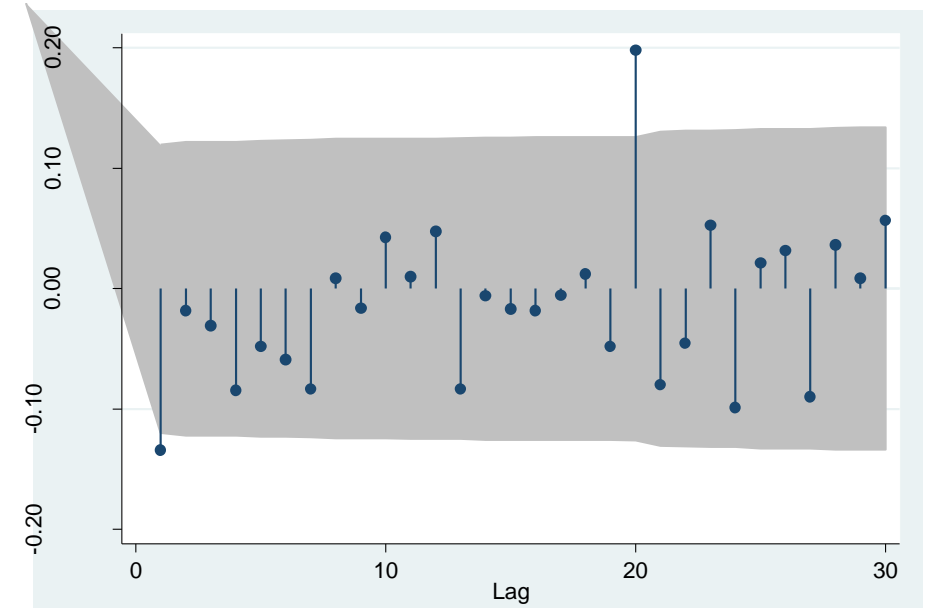

Fonte: Elaborado pelos autores com base nos resultados da pesquisa.

Por fim, para se chegar ao clímax de um modelo de previsão, para se obter os valores previstos, faz-se necessário integrar o modelo $\operatorname{AR}(4,12)$ MA $(4,12)$ representado na Equação 4, pois este está apresentado em primeira diferença, devido ao pressuposto de estacionariedade da modelagem ARIMA. Desse modo, para a previsão do consumo de energia elétrica para o período de junho de 2012 a Equação 03 pode ser transformada em:

$$
\begin{array}{r}
\left(\ln C_{2012.06}-\ln C_{2012.05}\right)=\delta+\alpha_{4}\left(\ln C_{2012.01}-\ln C_{2011.12}\right)+ \\
\alpha_{12}\left(\ln C_{2011.05}-\ln C_{2011.04}\right)+\gamma_{4} u_{2012.01}+\gamma_{12} u_{2011.05}+u_{2012.06}
\end{array}
$$

Nesta especificação o termo de erro $u_{2012.06}$ é presumido como sendo igual a zero. Desse modo a Equação 5 é transformada na Equação 6 a seguir:

$$
\begin{gathered}
\ln C_{2012.06}=\delta+\alpha_{4} \ln C_{2012.01}-\alpha_{4} \ln C_{2011.12}+\alpha_{12} \ln C_{2011.05}- \\
\alpha_{12} \ln C_{2011.04}+\gamma_{4} u_{2012.01}+\gamma_{12} u_{2011.05}+u_{2012.06}+\ln C_{2012.05}
\end{gathered}
$$

Assim, o modelo especificado na Equação 6 será utilizado para a previsão do consumo de energia elétrica para o mês de junho de 2012. De forma análoga, utiliza-se a Equação 03 para a derivação e especificação dos modelos de previsão para os meses subsequentes, ou seja, para os períodos de julho e agosto de 2012 . 
Então, após serem realizadas as devidas substituições dos valores reais nas equações derivadas da Equação 4, para os períodos de junho, julho e agosto de $2012^{11}$, encontram-se os valores previstos para o consumo, os quais estão representados na Tabela 12.

Através da análise da Tabela 12, é possível analisar o grau de exatidão das previsões realizadas, as quais variam de $96,89 \%$ a $98,70 \%$, com uma média de $97,46 \%$ de perfeição da previsão. Desse modo, encontram-se valores previstos para os meses de junho, julho e agosto de 2012, respectivamente de 15,65, 15,69 e 15,84 milhões de $M W h$, enquanto que os valores mensais, registrados pela Eletrobrás, foram respectivamente, 15,16, 15,19 e 15,63 milhões de $M W h$, indicando que os valores estimados superestimaram os valores reais.

Tais resultados são considerados estatisticamente consistentes apesar de se ter a base de dados mensais, em que as oscilações são mais acentuadas e menos previsíveis. Logo, os resultados da previsão confirmam que a modelagem ARIMA proposta é viável para realizar previsões de curtíssimo prazo.

Tabela 12: Previsão do consumo de energia elétrica para os meses de junho, julho e agosto de 2012.

\begin{tabular}{cccccc}
\hline Período & $\mathbf{l n C} *$ & $\begin{array}{c}\text { Valor } \\
\text { previsto }\end{array}$ & $\begin{array}{c}\text { Valor } \\
\text { realizado }\end{array}$ & $\Delta \%$ & $\begin{array}{c}\text { Grau de exatidão da } \\
\text { previsão em \% }\end{array}$ \\
\hline 2012.06 & 16,5662 & 15652866,40 & 15166000,00 & $-3,2102$ & 96,89 \\
2012.07 & 16,5687 & 15693303,65 & 15191000,00 & $-3,3066$ & 96,80 \\
2012.08 & 16,5781 & 15841237,91 & 15634000,00 & $-1,3256$ & 98,70 \\
& & & & Média: & $\mathbf{9 7 , 4 6}$ \\
\hline
\end{tabular}

* Efetua-se o antilogaritmo do valor estimado para $\ln C$ e assim encontra-se o valor previsto.

Fonte: elaborado pelos autores com base nos dados do modelo de previsão AR $(4,12) \operatorname{MA}(4,12)$.

\section{5) CONCLUSÃO}

No presente artigo, procurou-se estudar o setor elétrico brasileiro apresentando estimativas das elasticidades preço, elasticidade renda da demanda e elasticidade preço cruzado de energia elétrica do setor industrial

\footnotetext{
${ }^{11}$ Para não se tornar redundante as equações para os períodos de julho e agosto de 2012 não foram apresentas.
} 
do Brasil. Para isto, foram utilizados dados mensais do período de janeiro de 1990 a maio de 2012, em conjunto com testes estatísticos que representassem essas relações.

Com base nos dados percebesse que o consumo de energia elétrica industrial no Brasil aumentou 85,75\% de janeiro de 1990 a maio de 2012. Esse crescimento acompanhou o crescimento do PIB brasileiro no mesmo período, apresentando uma mesma tendência. Juntamente com o aumento do consumo, a tarifa cobrada sobre a utilização da energia elétrica também apresentou essa trajetória ascendente.

A matriz energética brasileira apesar de se originar de várias fontes, ainda se encontra concentrada em poucos setores. As fontes fósseis continuam significativas $(16,22 \%)$ e a hidroelétrica é a preponderante $(65,3 \%)$. Apesar de mais da metade da nossa matriz energética ser de fontes renováveis, a grande dependência de apenas uma fonte renovável, como a hidroelétrica deve ser analisada com cuidado, uma vez que no caso de secas sazonais, como as que vêm ocorrendo nos últimos anos pode comprometer a oferta de energia elétrica do país, fato que seria comprometedor considerando que a demanda apresenta um crescimento constante. Além disso, a alta carga tributária incidente sobre a tarifa de energia elétrica no Brasil contribui para tornar a tarifa brasileira uma das mais caras do mundo e a mais alta dos países do BRIC.

No presente trabalho foi estimada a demanda por energia elétrica para o Brasil para o período de janeiro de 1990 a maio de 2012 utilizando, primeiramente, um modelo de correção de erros vetoriais - VEC/MCE. Em tal etapa foi estimado o coeficiente de ajustamento $\left(\varepsilon_{t-1}\right)$, através do qual se verificou uma velocidade de ajustamento lenta, da ordem de $7 \%$ a cada período. A conclusão disto é que desequilíbrios de curto prazo são reduzidos lentamente. $\mathrm{Na}$ análise da função de resposta ao impulso tem-se que variações não antecipadas na renda e no preço do bem substituto (petróleo) têm impactos positivos na demanda de energia elétrica industrial. Enquanto que os efeitos de impulsos nos preços não são sentidos, refletindo os efeitos de uma demanda altamente inelástica.

As elasticidades encontradas foram de encontro com a teoria econômica e com trabalhos realizados por outros autores. Ou seja, restou confirmada a hipótese de que a energia elétrica é um bem normal, com demanda inelástica e funciona como um bem substituto em relação ao petróleo como fonte de energia para as indústrias nas quais é possível tal mobilidade. A elasticidade renda da demanda se mostrou significativa e estatisticamente maior que zero 
(0,4490). A elasticidade preço da demanda se mostrou inelástica (-0,0066), o que significa que a quantidade demandada é relativamente pouco sensível às variações do preço. Por outro lado, a elasticidade-preço cruzada da demanda $(0,1868)$ mostrou que o petróleo é um bem substituto para a energia elétrica usada no setor industrial.

Na sequência foram feitas as estimativas da demanda de energia elétrica de junho a agosto de 2012 utilizando o modelo ARIMA, o qual apresentou uma média de 97,46\% de perfeição da previsão, mostrando-se viável para realizar previsões de curtíssimo prazo. Nesse sentido, os resultados obtidos fornecem informações sobre a tendência do mercado de energia elétrica em análise, não devendo ser interpretados como resultados finais, uma vez que esse processo de escolha de modelos pode variar de acordo com os critérios adotados pelo pesquisador. Assim, essas tendências foram captadas pelas previsões realizadas, sendo convertidas em valores previstos.

Diferente de outros trabalhos apresentados, no presente estudo utilizouse dados mensais, o que melhora a capacidade de captação das possíveis volatilidades do setor. Da mesma maneira o poder de exatidão das previsões para os três próximos períodos mostrou-se muito alto, confirmando, portanto, que a modelagem ARIMA proposta é viável para realizar as previsões propostas. Nesse sentido, a consecução da presente pesquisa contribui com o debate acadêmico acerca de estudos que tem como foco este importante setor da economia brasileira, sendo de suma importância para o mesmo, por suprira carência de estudos recentes sobre a demanda industrial por energia elétrica no Brasil. 


\section{REFERÊNCIAS}

ANDRADE, L. C. M.; FLAUZINO, R. A.; SILVA, I. N. Sistemas Fuzzypara Previsão de Demanda de Energia Elétrica no Curtíssimo Prazo.Proceedings of the 9th Brazilian Conference on Dynamics Control and their Applications. Serra Negra/SP jun. 2010. Disponível em: <http://www.sbmac.org.br/dincon/trabalhos/PDF/control/67803.pdf>. Acesso em 16 nov. 2011.

ANDRADE, T. A.; LOBÃO, W. J. A. Elasticidade Renda e Preço da Demanda Residencial de Energia Elétrica no Brasil. Texto para Discussão no 489, Instituto de Pesquisa Econômica e Aplicada, Rio de Janeiro, jun. 1997. Disponível em: <http://www.pro.poli.usp.br/graduacao/backup/disciplinas-em andamento/pro2208_7/td0489.pdf>. Acesso em 16 nov. 2011.

ANEEL. Agência Nacional de Energia Elétrica.Brasília, Distrito Federal, 2012. Disponível em: 〈http://www.aneel.gov.br/>. Acesso em 16 out. 2012.

BUENO, R. de L. da S.Econometria de Séries Temporais. São Paulo, Ed. CENGAG Learning, 2008.

CASTRO, N. J. de; ROSENTAL, R.Nova tendência para a Elasticidade-Renda da Demanda de Energia Elétrica no Brasil. IFE - Informativo Eletrônico do Setor Elétrico - n. ${ }^{\circ} 2.215$ de 3 de mar. 2008. Disponível em: <www.brazilink.org/tiki-download_file.php?fileId=112>. Acessoem 16 nov. 2011.

ENDERS, W, Applied Econometric Time Series.Jonh Willey \& Sons, Inc., 2010.

EPE. Empresa de Pesquisa Energética.Projeção da Demanda de Energia Elétrica para os próximos 10 anos (2011-2020). Nota Técnica DEA 03/11. Rio de Janeiro, fev. 2011. Disponível em: <http://www.epe.gov.br/mercado/Documents/S\%C3\%A9rie\%20Estudos\%20de\%20Energia/20 110222_1.pdf>. Acesso em 16 nov. 2011.

GUJARATI, D. N.; PORTER, D. C. Econometria Básica. Rio de Janeiro: 5a Ed. AMGH Editora Ltda, 2011.

FIRJAN. Quanto custa a energia elétrica para a indústria no Brasil?Federação das Indústrias do Estado do Rio de Janeiro. Rio de Janeiro, No 8 , agosto, 2011.

HAMILTON, J. D. Time series analysis. 1. Ed. New Jersey: Princeton University Press, 1994.

IBGE. Instituto Brasileiro de Geografia e Estatística. Rio de Janeiro, 2011. Disponível em <http://www.ibge.gov.br>; Acessado em: 15 nov. 2011.

INFRAESTRUTURA. Anuário Exame.São Paulo: Editora Abril, 2009. 
IPEA. Instituto de Pesquisa Econômica Aplicada.Rio de Janeiro, 2011.Disponível em: <http://www.ipea.gov.br/portal/>. Acesso em: 1 nov. 2011.

IRFFI, G.; CASTELAR, I.; SIQUEIRA, M. L.; LINHARES, F. C.;Previsão da demanda por energia elétrica para classes de consumo na região Nordeste, usando OLS dinâmico e mudança de regime.Economia Aplicada, vol. 13 nº 1. Ribeirão Preto Jan./Mar. 2009.

LEITE, S. Q.Projeções para a demanda por energia elétrica no Brasil, 2006-2015. Dissertação de Mestrado Profissionalizante apresentada ao Programa de Pós-Graduação em Economia das Faculdades Ibmec. Rio de Janeiro, 2006.

MATOS, O. C. de. Econometria Básica. 3a ed. São Paulo: Atlas S.A., 2000.

MATTOS, L. B. Uma Estimativa da Demanda Industrial de Energia Elétrica no Brasil: 19742002. Organ. Rurais Agroind., Lavras, v. 7, n. 2, p. 238-246, 2005.

PINDYCK, R. S.; RUBINFELD, D. L. Econometria. 1ª ed. Rio de Janeiro: Elsevier, 2004.

SCHMIDT, C. A. J.; LIMA, M. A. M.A Demanda por Energia Elétrica no Brasil.RBE Rio de Janeiro 58(1):67-99 jan/mar 2004.

SIQUEIRA, M. L.; HOLLANDA, H.A Demanda Setorial por Energia Elétrica no PósRacionamento de 2001.Previsões de Longo Prazo para o Nordeste Brasileiro. Trabalho Submetido ao X Encontro Regional de Economia. [S.1.: s.n.], mai. 2005. Disponível em: <http://www.bnb.gov.br/content/aplicacao/ETENE/Anais/docs/mesa12_texto1.pdf>. Acesso em 16 nov. 2011.

VARIAN, H. R. Microeconomia: princípios básicos. $7^{\mathrm{a}}$ ed. Rio de Janeiro: Campus,2006. 